\title{
Herpes Simplex Type 2 Pneumonia
}

Edenilson Eduardo Calore
Emílio Ribas Institute, Biomedical Institute - São Paulo University, SP, Brazil

\begin{abstract}
Extensive reviews of pulmonary infections in AIDS have reported few herpetic infections. Generally these infections are due to Herpes simplex type 1. Pneumonia due to herpes type 2 is extremely rare. We describe a 40 year-old HIV positive woman who complained of fever, cough and dyspnea for seven years. She had signs of heart failure and the appearance of her genital vesicles was highly suggestive of genital herpes. Echocardiography showed marked pulmonary hypertension, right ventricular hypertrophy and tricuspid insufficiency. After a few days of hospitalization she was treated with Aciclovir and later with Ganciclovir. An open pulmonary biopsy revealed an interstitial inflammation, localized in the alveolar walls. Some pulmonary arteries had widened walls and focal hyaline degeneration. Immunohistochemistry indicated that the nuclei had herpes simplex virus type 2 in many endothelial cells (including vessels with widened walls), macrophages in the alveolar septa and pneumocytes. There was clinical improvement after treatment for herpes. We concluded that as a consequence of herpes infection, endothelial involvement and interstitial inflammation supervene, with thickening of vascular walls and partial obliteration of the vessel lumen. A direct consequence of these changes in pulmonary vasculature was pulmonary hypertension followed by heart failure.

Key Words: Herpes pneumonia, AIDS, opportunistic infections, pulmonary hypertension, herpes simplex.
\end{abstract}

Herpetic tracheobronchitis and pneumonia are rare complications of immunodeficient patients. Certain groups of subjects, including neonates, cancer patients, organ transplant recipients, and patients with cell mediated immunity deficiency are at increased risk for herpetic pneumonia [1]. Conditions eventually associated with herpes simplex are burns [2], postthoracotomy [3], acute myeloblastic leukemia [4] and necrotizing pneumonia in leptospirosis [5]. On the other hand, the viral agent that most frequently causes early neonatal pneumonia is herpes simplex [6]. Herpes pneumonia rarely occurs in immunocompetent individuals [7].

Received on 03 June 2002; revised 08 December 2002.

Address for correspondence: Dr. Edenilson Eduardo Calore, M. D., Ph.D. Emílio Ribas Institute - Pathology Section. Av. Dr. Arnaldo, 165 - 01246-902 - São Paulo - SP, Brazil. Fax: (55)(11)37511811.E-mail: calore@ sti.com.br

The Brazilian Journal of Infectious Diseases 2002;6(6):305-308 (C) 2002 by The Brazilian Journal of Infectious Diseases and Contexto Publishing. All rights reserved. 1413-8670
Acquired Immunodeficiency Syndrome (AIDS) epidemics have resulted in the emergence of a great number of opportunistic infections as well as increased incidence of serious diseases, such as tuberculosis (Tb) and other mycobacterioses that are now a public health problem, even in industrialized countries. Less frequently, unusual infective agents, such as free-living ameba or Rodococcus equi have been diagnosed in AIDS patients [8,9].

Oral and genital herpetic lesions are frequently observed in immunodeficient patients, particularly patients with AIDS. Other possible sites of Herpetic infections in AIDS are the esophagus and the central nervous system. However, herpes pneumonia is a rare condition. Extensive reviews of pulmonary infections in AIDS have reported few herpetic infections. Afessa [10] studied 1,225 consecutive hospital admissions and found only one case of herpes simplex pneumonia. Generally these infections are due to herpes simplex type 1 . Pneumonia due to herpes type 2 are extremely rare [11]. 
We studied a case of pneumonia due to herpes simplex type 2 , associated with pulmonary hypertension and heart failure.

\section{Case Report}

A 40 year-old woman, who has been HIV positive for seven years (ex-drug-addict) complained of fever, coughing and dyspnoea. At examination she had slight systemic arterial hypertension, cyanosis and edema of the lower limbs. She also had genital vesicles with symptoms highly suggestive of genital herpes. Morbid antecedents: systemic arterial hypertension, hepatitis $\mathrm{B}$ and $\mathrm{C}$ and pulmonary tuberculosis. She had used anti-retroviral drugs for six months; had anemia $(\mathrm{Hb}+10.8)$, and leucopenia with lymphopenia $(3,100$ leucocytes with $88 \%$ neutrophils), and $\mathrm{CD}_{4}^{+} 164$. She had renal failure $(U=99$ and $C=2.5)$. Lactic dehydrogenase $=685$. The patient was transferred to an Intensive Care Unit where she was maintained on mechanical ventilation. Blood gases were $\mathrm{pCO}_{2}=70$; $\mathrm{pCO}_{2}=42$ and $\mathrm{pH}=7.2$. Echocardiography indicated marked pulmonary hypertension (calculated pressure in the pulmonary artery $=90 \mathrm{mmHg}$ ), right ventricular hypertrophy and tricuspid insufficiency. At the hospitalization she was treated with Sulpha and cephalosporin. After few days she was treated with Aciclovir and subsequently with Ganciclovir.

An open pulmonary biopsy revealed a predominant interstitial inflammation in the alveolar walls. The alveolar septa were widened and edematous with lymphocytes and histiocytes. Hyaline alveolar membranes of proteinaceous material, which reflects alveolar damage, were eventually observed. There were many macrophages with clear and vacuolated cytoplasm in the lumen of the alveoli. Some endothelial cells had slightly enlarged and hyperchromatic nuclei. Some pulmonary arteries had widened walls and focal hyaline degeneration. Real obliterative endarteritis was eventually observed. Immunohistochemical tests for cytomegalovirus, respiratory syncytial virus and herpes simplex type 1 virus were negative. herpes simplex type 2 virus antigen was observed on the nuclei in many endothelial cells (including vessels with widened walls), macrophages in the alveolar septa and pneumocytes. Figures 1 to 6 illustrate these findings.

After treatment there was clinical improvement.

\section{Discussion}

Pulmonary opportunistic infections are one of the most important causes of morbidity and mortality in AIDS. The most frequent infectious agents are Pneumocystis carinni, Mycobacterium tuberculosis and cytomegalovirus. Less frequently other agents, such as Rodococcus equi, are causative agents of pneumonia.

Experimental studies have suggested that herpes simplex pneumonia induces an inflammation that is more dangerous than the direct cytopathic effects. In a study of secondary diffuse interstitial pneumonia, Oda [12], observed that classical herpetic inclusion bodies in lungs are not always associated with viral infection, which confirms the experimental studies of Adler et al. (1997) [13].

The few cases of herpetic pneumonia described in literature are caused by herpes simplex type 1 . We have found only one description of herpes simplex type 2 pneumonia. Although we observed characteristic genital lesions of herpes, the diagnosis of herpes pneumonia was performed only after lung biopsy and immunohistochemical tests. The most important symptoms of this patient were pulmonary hypertension and heart failure. The pathological studies revealed endothelial involvement and interstitial inflammation due to herpes simplex type 2 pneumonia. As a result of vasculitis, thickening of vascular walls and partial obliteration of vascular vessels, (changes in pulmonary vasculature) provoke pulmonary hypertension, followed by heart failure. Therefore, heart failure was probably indirectly caused by herpes simplex type 2 pneumonia.

The clinical improvement of this patient after treatment with anti-viral drugs is another indication that her symptoms were due to herpetic pneumonia, which supports the hypothesis that pulmonary hypertension was due to Herpetic infection. 


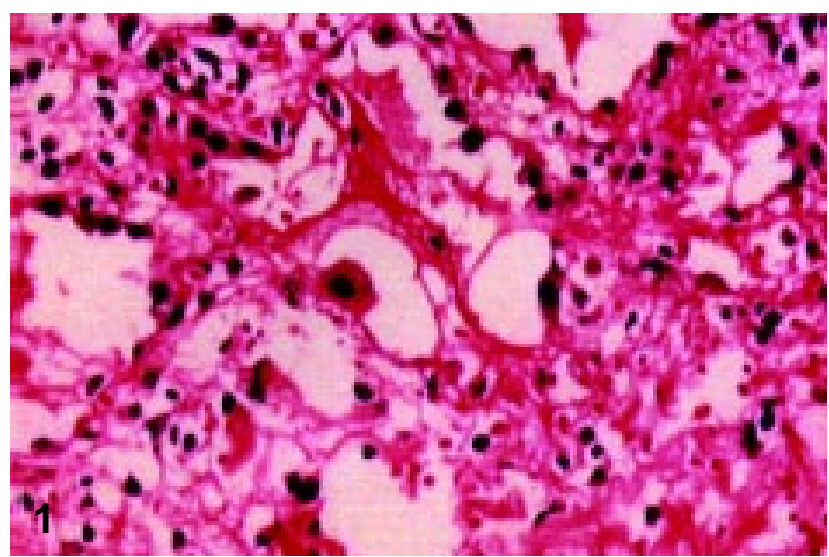

Reactive endothelial cells with hyperchromatic nuclei. Haematoxilin-eosin (x200).

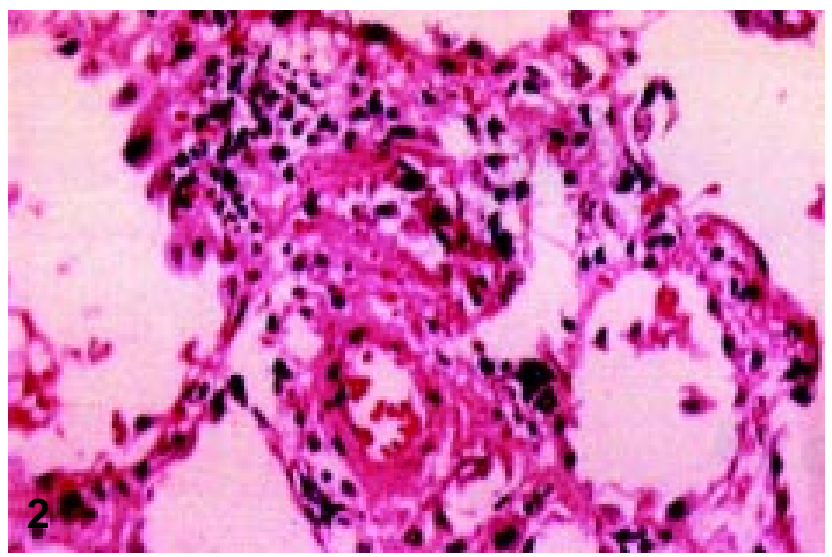

Obliterative endarteritis and lymphocytes in interstitium. Note some interstitial cells (presumable macrophages) that express Herpes simplex type 2 antigen. Immunohistochemical reaction for Herpes virus type 2 (x200).

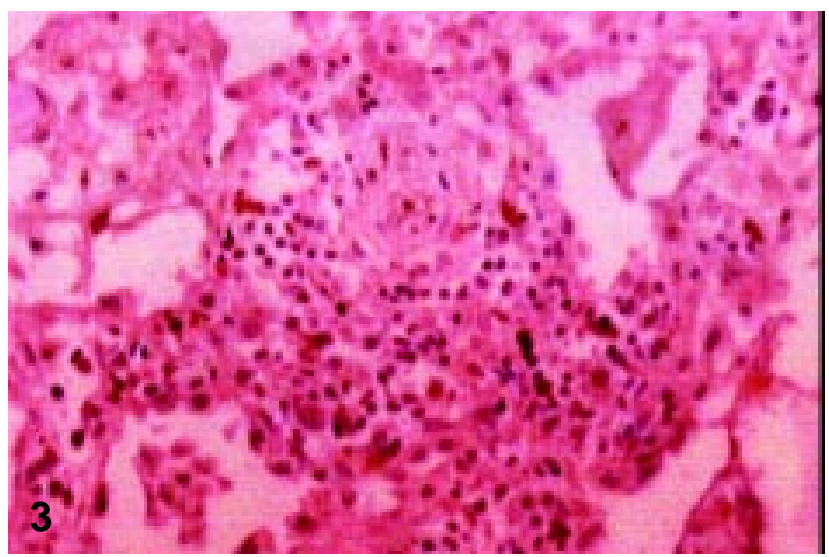

Thickness of a vascular wall. Note some interstitial cells positive for herpes simplex type 2 . Immunohistochemical reaction for herpes virus type 2 (x200).

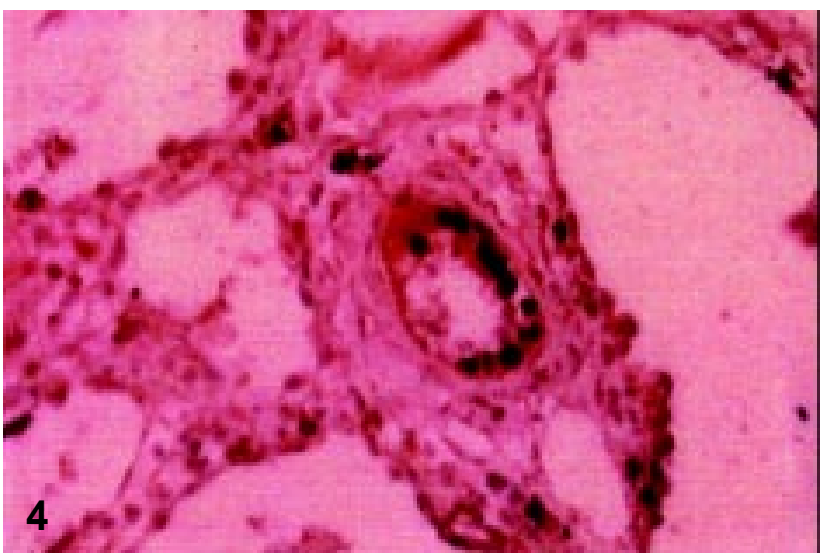

Vasculitis and endothelial cells with hyperchromatic nuclei suggesting viral nuclear inclusions - Haematoxilin-eosin staining (x200).

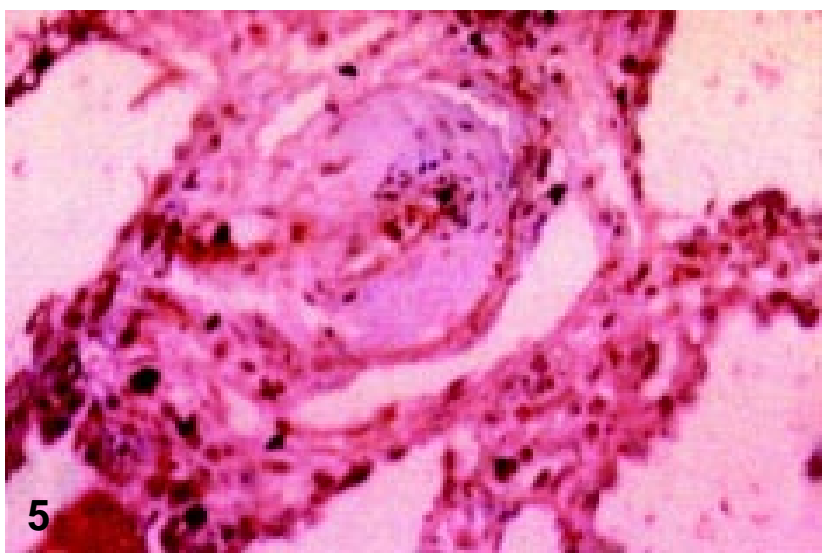

Many endothelial cells containing herpes antigens. The lumen of this vessel is partially obliterated by these cells. Immunohistochemical reaction for herpes virus type 2 (x200).

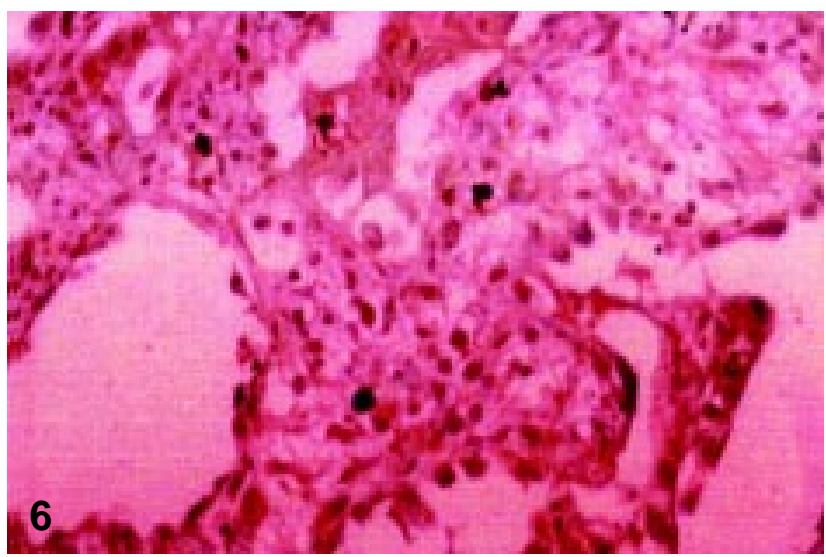

Edema and lymphocytic and macrophage reaction in the interstitium. Note that some cells are positive for herpes simplex type 2 antigen. Immunohistochemical reaction for herpes virus type 2 (x200). 


\section{References}

1. Feldman S., Stokes D.C. Varicella zoster and herpes simplex virus pneumonias. Semin Respir Infect 1987 Jun;2(2):84-94.

2. Byers R.J., Hasleton O.S., Quigley A., et al. Pulmonary herpes simplex in burns patients. Eur Respir J 1996;9(11):2313-7.

3. Camazine B., Antkowiak J.G., Nava M.E., et al. Herpes simplex viral pneumonia in the postthoracotomy patient. Chest 1995; 108(3):876-9.

4. James E., Robinson L., Griffiths P.D., Prentice H.G. Acute myeloblastic leukaemia presenting with herpes simplex type-1 viraemia and pneumonia. Br J Haematol 1996;93(2):401-2.

5. Bek M., Koppl H., Schwarzkopf G., Freudenberg N. Weil's disease and necrotizing herpes pneumonia as the cause of death of a 39-year-old sewage drain worker. Pathologe 1996; 17(6):471-6.

6. Albertini M. Neonatal pneumonia. Arch Pediatr 1998;5 Suppl 1:57s-61s.

7. Martinez E., de Diego A., Paradis A., et al. Herpes simplex pneumonia in a young immunocompetent man. Eur Respir J 1994; 7(6): 1185-8.

8. Calore E.E., Cavaliere M.J., Calore N.M.P. Cerebral amebiasis in Acquired Immunodeficiency Syndrome. Acta Neurol Belgica 1997;97:248-50.

9. Caterino-de-Araújo A., Santos-Fortuna E., ZandonMeleiro M.C., et al. Detection of the 20-kDA virulenceassociated antigen of the Rhodococcus equi in malakoplakia-like lesion in pleural tissue obtained from an AIDS patient. Pathol Res Pract 2000; 196 : 321-7.

10. Afessa B.M.D. Mycobacterial and nonbacterial pulmonary complications in hospitalized patients with human immunodeficiency virus infection: A prospective, cohort study. BMC Pulm Med 2001;1(1):1.

11. Furrer H., Meister F., Malinverni R. Herpes simplex type 2 pneumonia in an AIDS patient. Schweiz Med Wochenschr 1989;119(37):1275-8.

12. Oda Y., Okada Y., Katsuda S., Nakanishi I. Immunohistohemical study on the infection of herpes simplex virus, human cytomegalovirus and Epstein-Barr virus in secondary diffuse interstitial pneumonia. Hum Pathol 1994;25(10): 1057-62.

13. Adler H., Beland J.L., Del-Pan N.C., et al. Supreession of herpes simplex virus type-1 (HSV-1)-induced pneumonia in mice by inhibition of inducible nitric oxide synthase (iNOS, NOS2). J Exp Med 1997;5;185(9):1533-40. 